\title{
Measurement equivalence and feasibility of the EORTC QLQ-PR25: paper-and-pencil versus touch-screen administration
}

\author{
Yu-Jun Chang ${ }^{1,2}$, Chih-Hung Chang ${ }^{3,4}$, Chiao-Ling Peng ${ }^{1}$, Hsi-Chin Wu ${ }^{5,6}$, Hsueh-Chun Lin ${ }^{7}$, Jong-Yi Wang ${ }^{8}$, \\ Tsai-Chung Li $i^{3,9}$, Yi-Chun Yeh ${ }^{9}$ and Wen-Miin Liang ${ }^{1,3,9^{*}}$
}

\begin{abstract}
Objective: We assessed the measurement equivalence and feasibility of the paper-and-pencil and touch-screen modes of administration of the Taiwan Chinese version of the EORTC QLQ-PR25, a commonly used questionnaire to evaluate the health-related quality of life (HRQOL) in patients with prostate cancer in Taiwan.

Methods: A cross-over design study was conducted in 99 prostate cancer patients at an urology outpatient clinic. Descriptive exact and global agreement percentages, intraclass correlation, and equivalence test based on minimal clinically important difference (MCID) approach were used to examine the equity of HRQOL scores between these two modes of administration. We also evaluated the feasibility of computerized assessment based on patients' acceptability and preference. Additionally, we used Rasch rating scale model to assess differential item functioning (DIF) between the two modes of administration.

Results: The percentages of global agreement in all domains were greater than $85 \%$ in the EORTC QLQ-PR25. All results from equivalence tests were significant, except for Sexual functioning, indicating good equivalence. Only one item exhibited DIF between the two modes. Although nearly $80 \%$ of the study patients had no prior computer-use experience, the overall proportion of acceptance and preference for the touch-screen mode were quite high and there was no significant difference across age groups or between computer-use experience groups.

Conclusions: The study results showed that the data obtained from the modes of administration were equivalent. The touch-screen mode of administration can be a feasible and suitable alternative to the paper-and-pencil mode for assessment of patient-reported outcomes in patients with prostate cancer.
\end{abstract}

Keywords: Health-related quality of life, Prostate cancer, EORTC QLQ-PR25, Equivalence, Feasibility, Cross-over design, Touch-screen, Paper-and-pencil

\section{Introduction}

The proper use of patient-reported outcome (PRO) measurement in clinical settings has become increasingly important to obtain more comprehensive information to guide clinical decision-making, treatment planning, and clinical management [1]. Traditionally, PRO data are collected through face-to-face interviews or patient self-report to paper-based questionnaires, which is labor

\footnotetext{
* Correspondence: wmliang@mail.cmu.edu.tw

${ }^{1}$ Graduate Institute of Public Health, China Medical University, Taichung, Taiwan

${ }^{3}$ Graduate Institute of Biostatistics, China Medical University, No.91 Hsueh-Shih Road, Taichung 40402, Taiwan

Full list of author information is available at the end of the article
}

intensive and time consuming. With the emergence of computer technology, electronic methods of data collection (e.g., touch-screen response or interactive voice response) are becoming more popular and viable alternatives to conventional surveys carried out in clinical practice [1-3].

Electronically administered questionnaires allow data to be automatically entered real time into a database, after which the score is immediately calculated; thus, data coding errors and the workload of health professionals are reduced [3,4]. The time required by the patient to complete the electronically administered questionnaire such as electronic patient-reported outcome (ePRO) questionnaire, 
is also reduced for routine clinical practice [3,5]. In addition, increased use of the ePRO questionnaires in clinical assessments may promote integration of PRO and clinical information. Once patients have completed the ePRO questionnaires during their clinic visits, their item responses will be automatically scored and summarized for potential clinical use with other patient-related information. The integrated results are readily available in easily interpretable reports that can be viewed together by the clinician and their patient during clinical encounter. Therefore, the process can enhance the efficiency and quality of healthcare and patient-physician communications $[3,6,7]$. Nonetheless, the equivalence of the ePRO version and its original paper-and-pencil version should be thoroughly evaluated, and the patient preference and acceptance should also be examined before shifting from paper-and-pencil data to ePROs without demonstrating its feasibility $[6,8]$. Some studies have examined and validated the measurement equivalence of paper-and-pencil-based version and touch-screen computer-based version; the results showed that the data collected from paper- and computeradministered PROs were very similar and the touch-screen version was well accepted by most subjects [6-10].

Prostate cancer is a common disease among men in many Western countries and developed Asian countries. The standardized incidence in Taiwan (adjusted by the 2000 world population) has increased from 1.86 per 100,000 men in 1979 to 28.77 per 100,000 men in 2010 [11]. Moreover, long-term survival results have shown that health-related quality of life (HRQOL) has become an important outcome measure in different clinical settings [12]. The European Organization for Research and Treatment of Cancer (EORTC) Quality of Life Study Group developed the EORTC QLQ-PR25, a 25-item questionnaire designed for use among patients with localized and metastatic prostate cancer, is a commonly used tool to assess HRQOL in patients with prostate cancer. It includes four domains that assess urinary symptoms, bowel symptoms, treatmentrelated symptoms, and sexual activity and functioning. The results of international field validation have been published in 2008 [13]. The paper-and-pencil versions of the EORTC QLQ-PR25 have satisfactory reliability and validity [12-14]. In this study, we used the Taiwan Chinese version of the EORTC QLQ-PR25 questionnaire published by Chie et al. in 2010 [12]. It was also shown to be reliable and valid to assess HRQOL using the modern test theory approach in our previous study [15]. To the best of our knowledge, the psychometric properties and feasibility of the touch-screen version of this questionnaire for prostate cancer patients have not been well established, and no data have been reported in Taiwan. Therefore, in this study we sought to assess the measurement equivalence and feasibility of the paper-and-pencil and touch-screen versions of the EORTC QLQ-PR25 in patients with prostate cancer in Taiwan.

\section{Methods}

\section{Research design and data collection}

A randomized cross-over design was used in this study. A total of 107 prostate cancer patients in various stages of illness and treatment were enrolled from September 2008 to October 2009 in the Department of Urology outpatient clinic of China Medical University Hospital in Central Taiwan. Patients who could not read, speak, or write Chinese were excluded. All patients provided written informed consent. The study procedures were approved by the Institutional Review Board of the China Medical University Hospital.

Initially, 107 patients with prostate cancer were enrolled and randomly assigned into one of the two study groups, with 54 patients in the paper-and-pencil-version-first group (denoted as paper/touch-screen group) and 53 patients in the touch-screen-version-first group (denoted as touch-screen/paper group). Each patient was asked to complete both versions of the questionnaires. Patients in the paper/touch-screen group were given the paper-and-pencil version of the questionnaire first, followed by the touch-screen version after a 120-minute interval. By contrast, the touch-screen/paper patients were given the touch-screen questionnaire first, followed by the paper-and-pencil version 120 minutes later. Once the patients finished the first mode of administration, they were led to the education room to watch health education videos. This was to dilute the memory of their responses to the questions from the first administration as the same set of question were asked at the second administration. After completing each questionnaire for the two modes of administration, each patient was asked to answer a usability and feasibility questionnaire to indicate their preference and acceptance of the touch-screen version of the questionnaire. Ninety-nine patients successfully completed both assessments and eight patients had to leave early and did not complete the whole procedure. The study scheme is shown in Figure 1.

\section{HRQOL measures}

The prostate-specific module EORTC QLQ-PR25 is a selfadministered questionnaire that includes 4 subscales for assessment of Urinary symptoms ( 9 items, labeled US31-US39), Bowel symptoms (4 items, BS40-BS43), Hormonal treatment-related symptoms (6 items, TS44-TS49), and Sexual activity and function (6 items, SX50-SX55). In this study, no patients reported using incontinence aid, therefore, the item (US38, "Has wearing an incontinence aid been a problem for you? Answer this question only if you wear an incontinence aid.") was excluded from the Urinary symptom domain for analysis. Each of the 24 retained items was scored from 1 to $4(1=$ "Not at all", $2=$ "A little", 3 = "Quite a bit", and 4= "Very much") [13]. Domain scores of the EORTC QLQ-PR25 were linearly 


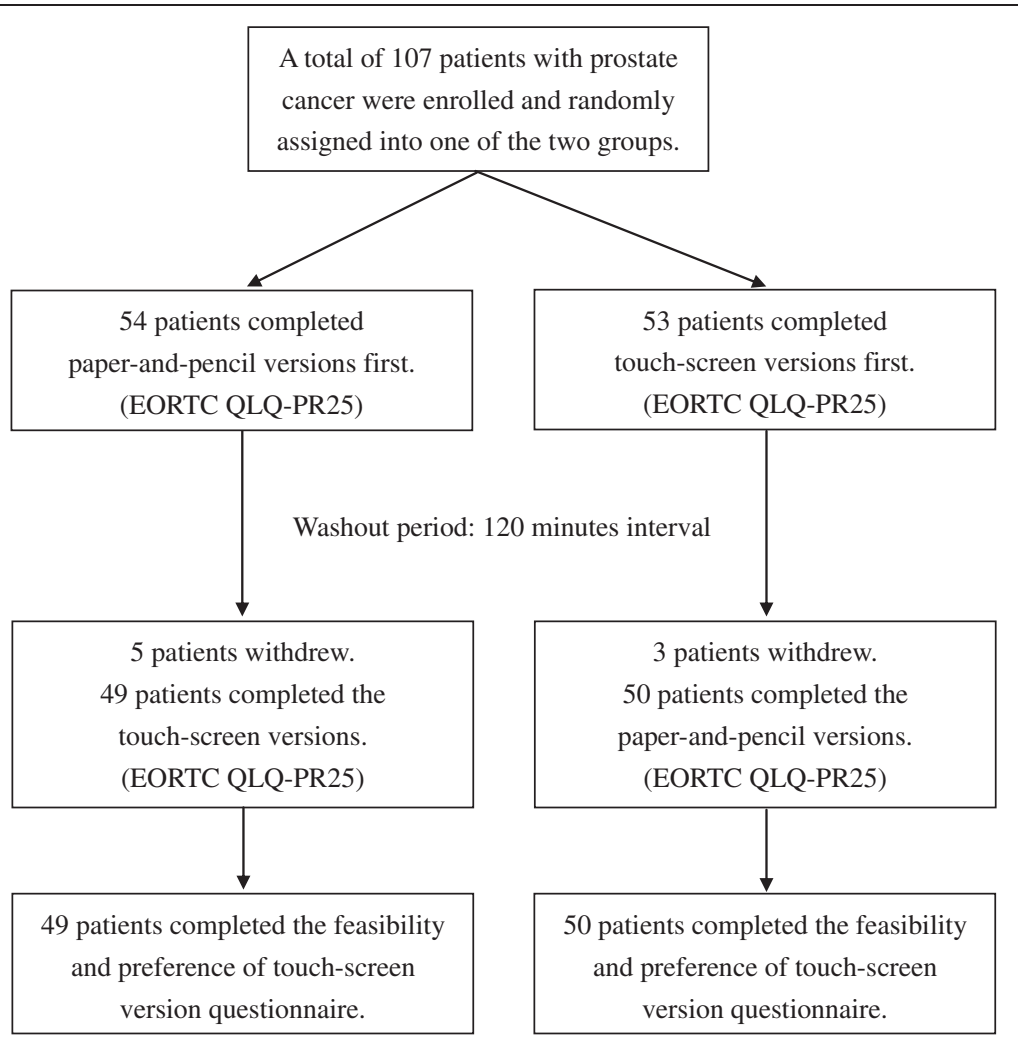

Figure 1 Study structure in the study.

transformed to a 0 to 100 scale based on its scoring manual, which requires answering at least half of the total number of items in the domain. Higher scores reflected either more symptoms (e.g., Urinary, Bowel, and Hormonal treatment-related symptoms) or higher levels of functioning (e.g., Sexual activity and function) [16].

\section{Setting of the touch-screen version}

The touch-screen version was developed by a team of physicians specializing in prostate cancer, technicians with expertise in system design and programming, epidemiologists, and statisticians. Java software was used to develop the system using an Oracle database [17].

\section{Statistical analysis}

Descriptive statistics for continuous variables are presented as means and standard deviations, whereas categorical variables are presented as frequencies and proportions. We assessed the differences in demographic characteristics and time to complete the questionnaire between the two study groups using independent $t$-test for continuous data and Chi-square or Fisher's exact test for categorical data. And a paired $t$-test was used to compare the administration time between two modes.

We used a mixed model to assess the quality of the cross-over design, wherein the dependent variable was the domain score and the main independent variables were administration order (order effect), mode type (mode effect), and their interaction (carry-over effect). A random effect accounted for the covariance structure induced by the repeated measures. Gender and age effects were also included for adjustment of confounding effect. After testing the mode-by-order interaction, we fit the model again without interaction term (i.e., main-effect model) if the carry-over effect is not statistically significant at 5\% level. No significant carry-over effect in the interaction model and no significant order effect in the main-effect model indicated methodologically appropriate for the cross-over design.

The agreement of scores between the paper and the computerized administrations was assessed at the individual patient level. "Exact agreement" referred to as patients who provided the same responses to individual questions on both paper-and-pencil- and touch-screen-administered questionnaires. "Global agreement" was defined as the proportion of agreement within one adjacent response category in either higher or lower direction [18]. We also used intraclass correlations (ICC) calculated from a random-effects mixed model and interpreted the results based on criteria proposed by Bartko et al. [19] and Stokdijk et al. [20] as follows: an ICC $<0.40$ indicated poor agreement, 0.40 to 0.59 indicated moderate 
Table 1 Demographic characteristics and time for completion of questionnaires of the two groups of prostate cancer patients

\begin{tabular}{|c|c|c|c|}
\hline & $\begin{array}{l}\text { Paper/Touch-screen } \\
\qquad(n=49)\end{array}$ & $\begin{array}{l}\text { Touch-screen/Paper } \\
\qquad(n=50)\end{array}$ & $p$-value \\
\hline \multicolumn{4}{|l|}{ Age (year) (N (\%)) } \\
\hline$<=65$ & $12(24.5)$ & $13(26.0)$ & $0.778^{b}$ \\
\hline $66-70$ & $11(22.5)$ & $14(28.0)$ & \\
\hline $71-75$ & $12(24.5)$ & $13(26.0)$ & \\
\hline$>75$ & $14(28.5)$ & $10(20.0)$ & \\
\hline Mean (SD) & $70.1(7.6)$ & $69.0(8.1)$ & $0.463^{\mathrm{a}}$ \\
\hline Education level (N (\%)) & & & $0.701^{\mathrm{b}}$ \\
\hline College or above & $14(29.8)$ & $13(26.0)$ & \\
\hline Senior high & $11(23.3)$ & $15(30.0)$ & \\
\hline Junior high & $11(21.4)$ & $7(14.0)$ & \\
\hline Primary school or less & $12(25.5)$ & $15(30.0)$ & \\
\hline \multicolumn{4}{|c|}{ Previous experience using computers (N (\%)) } \\
\hline Yes & $10(20.4)$ & $9(18.0)$ & $0.837^{b}$ \\
\hline No & $39(79.6)$ & $41(82.0)$ & \\
\hline \multicolumn{4}{|c|}{ Time for completion of questionnaires (min) ${ }^{d}$} \\
\hline Paper version (Mean (Range)) & $17.9(5.0 \sim 39.0)$ & $14.7(6.0 \sim 31.0)$ & $0.022^{\mathrm{a}}$ \\
\hline Touch-screen version (Mean (Range)) & $15.7(5.0 \sim 30.0)$ & $20.5(9.0 \sim 41.0)$ & $0.002^{\mathrm{a}}$ \\
\hline$p$-value & $0.516^{c}$ & $<0.001^{\mathrm{c}}$ & \\
\hline
\end{tabular}

${ }^{a} p$-value was evaluated by Independent $t$-test.

${ }^{\mathrm{b}} p$-value was evaluated by Chi-square test.

${ }^{c} p$-value was evaluated by Paired $t$-test.

${ }^{\mathrm{d}}$ Time for completion of the two questionnaire modes by four questionnaires, including the EORTC QLQ-C30, EORTC QLQ-PR25, IIEF-5

(International Index of Erectile Function), and IPSS (International Prostate Symptom Score).

agreement, 0.60 to 0.75 indicated good agreement, and $>0.75$ indicated excellent agreement. Highly positive ICCs indicate that paper and computer measures covary, and the mean and variability of the scores are similar [2].

In addition, we used a "Two One-Sided Test" procedure to determine whether the two administrations produce equivalent results. Equivalence testing is operationally different from the conventional method (e.g., independent $t$-test), which is mainly used to detect difference rather than equivalence. Equivalence testing refers to a trial wherein the primary objective is to show that the response to the novel intervention is as good as the response to the standard intervention. This procedure begins with attempting to demonstrate that they are equivalent within a practical, preset limit $\delta$ (i.e., $\left|\mu_{\text {paper }}-\mu_{\text {touch }- \text { screen }}\right|<\delta$ ), and sets a null hypothesis that the two mean values are not equivalent (i.e., $\mid \mu_{\text {paper }}-\mu_{\text {touch }}-$ screen $\mid \geq \delta$ ). The method we used is computationally identical to perform two one-sided $t$-tests with the following sets of hypotheses:
This premise is conceptually opposite to that of the conventional independent $t$-test procedure [21]. The occurrence of both rejections from two one-sided $t$-tests at $5 \%$ significant level indicates that the two modes are equivalent, which means that the difference between the groups is not more than a tolerably small amount (i.e., $\mid \mu_{\text {paper }}-\mu_{\text {touch }}-$ screen $\left.\mid<\delta\right)[22,23]$. This small amount of allowable difference is the margin that defines the "zone of indifference" where the interventions are considered equivalent [24]. In our analysis, we used a minimum clinically important difference (MCID) of 5 (i.e., $\delta=5$ ) based on previous studies $[25,26]$, as the tolerable amount to assess equivalence.

The required sample size for this study was based on the assumption that no clinical differences are present between the domain scores of the two administration modes under a cross-over design study. The MCID for each domain score of the EORTC QLQ-PR25 was set at a five-point score, and the standard error of the domain

$$
\begin{gathered}
\text { Left tail } \\
\left\{\begin{array} { c } 
{ H _ { 0 } : D = \mu _ { \text { paper } } - \mu _ { \text { touch-screen } } \leq - \delta } \\
{ H _ { 1 } : D = \mu _ { \text { paper } } - \mu _ { \text { touch-screen } } > - \delta }
\end{array} \quad \left\{\begin{array}{l}
H_{0}: D=\mu_{\text {paper }}-\mu_{\text {touch-screen }} \geq \delta \\
H_{1}: D=\mu_{\text {paper }}-\mu_{\text {touch-screen }}<\delta
\end{array}\right.\right.
\end{gathered}
$$


score was set at 8 based on the empirical data. The minimum sample size was estimated to be 80 using the statistical software PASS to detect an equivalence difference of 5 with $80 \%$ power and $5 \%$ type I error.

\section{Confirmation from modern measurement theory}

Rasch analysis, based on the modern measurement theory, has been shown to be a useful tool for the development of new PRO measures and in evaluating the measurement structure of existing PRO measures $[27,28]$. We used the rating scale model (RSM) to estimate difficulty calibration for each item. RSM, an extension of the dichotomous Rasch model, for polytomous items with ordered response categories was chosen as it is suitable for the items used in this study. In Rasch analysis, differential item functioning (DIF) can be used to examine item measurement invariance $[29,30]$. In this study, DIF, an item lacking equivalence in performance across the two groups or settings (e.g., paperand-pencil vs. touch-screen administration), was identified statistically by conducting an independent $t$-test on the difficulty calibration of each item. An item was said to exhibit DIF if the test was significant $(p<0.05)$ [31].

\section{Results}

\section{Demographic characteristics}

Table 1 shows the demographic characteristics of the study patients with prostate cancer. The mean age was 70.1 years in the paper-and-pencil/touch-screen group and 69 years in the touch-screen/paper-and-pencil group. The age range of patients was 57 years to 87 years. More than half of the participants graduated from high school. Approximately $80 \%$ of the patients had no experience using a computer. No statistically significant differences were observed for demographic characteristics between the two groups.

\section{Mixed model analysis}

We conducted the mixed model with two main effects (accounted for the mode effect and the order effect) and their interaction (accounted for the carry-over effect). No carry-over effects were found ( $p$-value $>0.05$ ). We then removed the interaction and reran the model. No order effect was observed ( $p$-value $>0.05)$. The results confirmed the quality of the cross-over design.

\section{Exact and global agreement analysis}

Table 2 shows the percentages of exact and global agreements for each domain. In the "urinary symptoms" domain that included 8 items, 791 paired responses $(8$ items $\times 99$ subjects - 1 missing pair) were noted. Out of 791, 629 paired responses were identical, which yielded an exact agreement percentage of $80 \%(=629 / 791)$. Our results showed that the percentages of exact agreement for all domains in EORTC QLQ-PR25 ranged from 61\% to $89 \%$.
Table 2 Exact and global agreements and ICC between touch-screen and paper-and-pencil modes

\begin{tabular}{|c|c|c|c|}
\hline EORTC QLQ-PR25 & $\begin{array}{c}\text { Exact } \\
\text { agreement }^{\mathrm{a}}\end{array}$ & $\begin{array}{c}\text { Global } \\
\text { agreement }^{\mathrm{b}}\end{array}$ & ICC \\
\hline Urinary symptoms (8 items) ${ }^{c}$ & 629/791 (80\%) & 778/791 (98\%) & 0.78 \\
\hline Bowel symptoms (4 items) & $351 / 396(89 \%)$ & $396 / 396(100 \%)$ & 0.72 \\
\hline $\begin{array}{l}\text { Treatment-related symptoms } \\
\text { (6 items) }\end{array}$ & $500 / 591(85 \%)$ & $574 / 591(97 \%)$ & 0.47 \\
\hline Sexual activity (2 items) & 142/196 (72\%) & 187/196 (95\%) & 0.61 \\
\hline Sexual functioning (4 items) ${ }^{d}$ & $92 / 151(61 \%)$ & 133/151 (88\%) & 0.45 \\
\hline
\end{tabular}

Number of total pairs $=$ number of subjects $\times$ number of items - number of missing pairs.

${ }^{a}$ Exact agreement (in\%): number of same response pairs/number of total pairs. ${ }^{\mathrm{b}} \mathrm{Global}$ agreement (in\%): number of within one-difference response pairs/number of total pairs.

In our study, there is no patients reported using incontinence aid, therefore, we deleted an item (US38 "Incontinence aid") from the Urinary symptom domain, resulting in 8 items remained in this domain.

${ }^{d}$ Sexual functioning items only apply to sexually active subjects. A total of 48 patients reported being sexually active. Meanwhile, forty-one missing pairs occurred in this domain.

The percentages of global agreement (i.e., the difference of scores of each paired responses was within one response category) ranged from $88 \%$ to $100 \%$.

\section{Intraclass correlation coefficient analysis}

The intraclass correlation coefficients (ICCs) ranged from 0.45 ("Sexual Function" domain) to 0.78 ("Urinary symptoms" domain) for all the domains in the EORTC QLQ-PR25 (Table 2), indicating moderate to excellent agreement for each domain between two modes.

\section{Equivalence test based on minimal important difference approach}

Table 3 shows the results of the domain scores and equivalence test based on the MCID approach for comparison of touch-screen and paper-and-pencil modes. Equivalence tests based on the MCID of 5 were used to assess the equivalent properties between the two modes and the results showed the measurement scales between two modes were equivalent for all domains except for Sexual functioning domain.

\section{DIF analysis}

Table 4 shows the results of DIF of Rasch analysis. No DIF was found for EORTC QLQ-PR25 for prostate cancer patients, except item 31 ("Urinary frequency during daytime"). In general, the measurement properties for each item were equivalent between the two modes.

\section{Acceptance and preference for touch-screen mode}

Table 5 shows the patients' views about the use of touchscreen questionnaire administration. Approximately 92\% of patients reported that the touch-screen questionnaire administration was easy to use and approximately $97 \%$ 
Table 3 Mean scores and equivalence test between touch-screen and paper-and-pencil modes

\begin{tabular}{|c|c|c|c|c|c|c|c|}
\hline \multirow{3}{*}{ EORTC QLQ-PR25 ${ }^{\mathrm{a}}$} & \multicolumn{2}{|c|}{ Paper-and-pencil } & \multicolumn{2}{|c|}{ Touch-screen } & \multicolumn{3}{|c|}{ Equivalence test $^{c}$} \\
\hline & Mean & SD & Mean & SD & $95 \% \mathrm{Cl}$ of & Left tail $^{\mathrm{e}}$ & Right tail $^{\mathrm{e}}$ \\
\hline & & & & & Mean difference ${ }^{d}$ & $\overline{p \text {-value }}$ & $p$-value \\
\hline Urinary symptoms (8 items) & 19.5 & 13.5 & 21.1 & 13.3 & -3.3 to 0.1 & $<0.001$ & $<0.001$ \\
\hline Bowel symptoms (4 items) & 5.3 & 8.6 & 5.7 & 7.9 & -1.5 to 1.0 & $<0.001$ & $<0.001$ \\
\hline Treatment-related symptoms ( 6 items) & 11.1 & 10.2 & 10.3 & 9.2 & -1.1 to 2.9 & $<0.001$ & $<0.001$ \\
\hline Sexual activity ( 2 items) & 19.4 & 20.2 & 20.2 & 20.8 & -5.0 to 2.3 & 0.048 & $<0.001$ \\
\hline Sexual functioning (4 items) $)^{b}$ & 62.3 & 22.0 & 61.8 & 20.9 & -5.7 to 9.5 & 0.076 & 0.415 \\
\hline
\end{tabular}

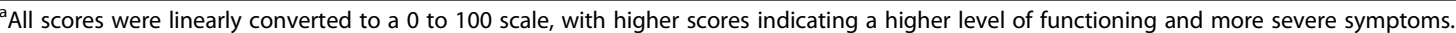

${ }^{\mathrm{b}}$ Sexual functioning items are conditional and only applicable to those being sexually active. A total of 48 patients reported being sexually active in this study.

'Based on the equivalence test using the score 5 as the tolerably difference.

${ }^{\mathrm{d}}$ Mean difference $=$ paper-and-pencil mean score - touch-screen mean score.

e Left tail significance indicates $\mu_{\text {paper }}-\mu_{\text {touch-screen }}>-5$. Right tail significance indicated $\mu_{\text {paper }}-\mu_{\text {touch-screen }}<5$. Therefore, both tails significance indicates

$\left|\mu_{\text {paper }}-\mu_{\text {touch-screen }}\right|<5$

thought the user interface was friendly. Approximately 92\% of patients stated that they liked using the touch-screen to complete the questionnaire. More than two-thirds $(67 \%)$ of the patients said they preferred using the touch-screen mode to fill out the questionnaire, while $30 \%$ preferred using the paper-and-pencil mode. Based on the results stratified by age, higher percentages of acceptance and preference were observed in patients $<=70$ years of age compared with patients aged $>70$ years. However, no statistically significant difference for each question was observed when the two age groups were compared. One hundred percent of prostate cancer patients with experience using a computer and $90 \%$ to $96 \%$ of patients without previous computer experience believed that the touch-screen mode was user friendly. Although nearly $80 \%$ of the prostate cancer patients had no computer experience, the overall percentages of acceptance and preference for the touch-screen mode was quite high and was non-significantly different compared with the results between the patients with and without computer experience.

\section{Discussion}

The measurement equivalence between paper-based versions and touch-screen versions of the questionnaires has been previously demonstrated in various diseases $[4,7,18,32]$, but to the best of our knowledge no data on the EORTC QLQ-PR25 in Taiwan have been reported. Our results showed the percentages of global agreement in all EORTC QLQ-PR25 domains were $>85 \%$. All results from equivalence tests were significant, indicating measurement equivalence, except for Sexual functioning domain. The results of measurement equivalence were confirmed using the modern test theory approach. Only one out of 24 items exhibited DIF between the two modes. The overall rate of acceptance and preference for the touch-screen mode were quite high.

In order to develop a computerized version of the EORTC QLQ-PR25 for use in this study, we completed a required user's agreement that permits us to use the questionnaire or for any change such as computerizing the questionnaire, and be held responsible for the quality of the measurement [33]. Permission was granted, per its policy, to allow us to use and migrate the paper form of the EORTC QLQ-PR25 to a tablet format for research purpose. Moreover, the U.S. Food and Drug Administration (FDA) released a PRO guidance in 2009, which suggested that a small randomized study is needed to provide evidence to confirm the new instrument's adequacy for any change of instrument such as changing an instrument from paper to electronic format [34].

In our randomized cross-over designs, subjects were randomized into one of the two following sequences: paper then touch-screen or touch-screen then paper. Therefore, each subject served as his own control. Cross-over trials were conducted within participant comparisons, whereas parallel designs were conducted between participant comparisons. The influence of confounding covariates and the majority of between-patient variation could be eliminated using a cross-over design [34,35]. In this study, our results from mixed model analysis showed that no mode-by-order interaction effect was present; thus, the carry-over effect did not exist. Moreover, when we refitted the main effect by interaction term removal, the order effect did not exist. These results showed that the cross-over randomized design in our study is methodologically appropriate. Fewer patients may be required in the cross-over design to attain the same level of statistical power and precision. Moreover, this design permitted opportunities of head-to-head trials, and subjects receiving multiple treatments can express preferences for or against particular treatments $[35,36]$.

In this study, we used intraclass correlation coefficients (ICCs), i.e., Pearson correlation coefficients when there were only two evaluations within a subject, to estimate the between-mode (touch-screen to paper; paper to touch-screen) test-retest reliability $(0.40-0.84$ for each item, $0.45-0.78$ for each domain, and 0.80 for total score), 
Table 4 Using Rasch analysis with rating scale model in differential item functioning analysis

\begin{tabular}{|c|c|c|c|c|c|c|c|c|}
\hline & \multirow{2}{*}{$\begin{array}{l}\text { EORTC QLQ-PR25 } \\
\text { Items ranked by difficulty }\end{array}$} & \multicolumn{2}{|c|}{ Paper } & \multicolumn{2}{|c|}{ Computer } & \multicolumn{3}{|c|}{ Differential item functioning } \\
\hline & & Difficulty & SE & Difficulty & $\mathrm{SE}$ & Difficulty & SE & $p$-value \\
\hline \multicolumn{9}{|c|}{ Urinary symptoms (US) $^{\mathrm{a}}$} \\
\hline US37 & Painful voiding (least frequent) & -3.055 & 0.369 & -2.790 & 0.321 & -0.266 & 0.489 & 0.588 \\
\hline US39 & Limitation of daily activities because of US & -1.035 & 0.250 & -1.306 & 0.251 & 0.271 & 0.354 & 0.445 \\
\hline US36 & Urinary incontinence & -0.199 & 0.225 & -0.349 & 0.226 & 0.150 & 0.319 & 0.638 \\
\hline US35 & Need to remain close to toilet & -0.149 & 0.223 & -0.346 & 0.226 & 0.197 & 0.318 & 0.536 \\
\hline US31 & Urinary frequency in daytime & 0.599 & 0.210 & 1.261 & 0.200 & -0.662 & 0.290 & 0.024 \\
\hline US34 & Sleep deprivation because of US & 1.118 & 0.206 & 0.766 & 0.207 & 0.352 & 0.292 & 0.229 \\
\hline US33 & Urinary urgency & 1.118 & 0.206 & 1.420 & 0.198 & -0.302 & 0.286 & 0.292 \\
\hline US32 & Nocturia (most frequent) & 1.537 & 0.204 & 1.301 & 0.199 & 0.236 & 0.285 & 0.410 \\
\hline \multicolumn{9}{|c|}{ Bowel symptoms (BS) ${ }^{\mathrm{a}}$} \\
\hline BS42 & Fecal blood (least frequent) & -0.656 & 0.413 & -0.839 & 0.404 & 0.183 & 0.578 & 0.752 \\
\hline BS41 & Fecal incontinence & -0.656 & 0.413 & -0.535 & 0.377 & -0.120 & 0.559 & 0.830 \\
\hline BS40 & Limitation of daily activities because of BS & 0.437 & 0.339 & 0.313 & 0.328 & 0.124 & 0.472 & 0.793 \\
\hline BS43 & Bloated feeling (most frequent) & 0.879 & 0.327 & 1.026 & 0.315 & -0.147 & 0.454 & 0.747 \\
\hline \multicolumn{9}{|c|}{ Treatment-related symptoms (TS) ${ }^{a}$} \\
\hline TS45 & Breast tenderness (least frequent) & -1.676 & 0.384 & -1.489 & 0.381 & -0.187 & 0.541 & 0.731 \\
\hline TS46 & Swelling in legs or ankles & -0.449 & 0.257 & -0.151 & 0.247 & -0.298 & 0.357 & 0.406 \\
\hline TS44 & Hot flushes & -0.384 & 0.253 & -0.812 & 0.301 & 0.429 & 0.393 & 0.277 \\
\hline TS47 & Bother due to weight loss & -0.035 & 0.234 & 0.284 & 0.221 & -0.320 & 0.322 & 0.322 \\
\hline TS48 & Bother due to weight gain & 0.668 & 0.200 & 0.515 & 0.209 & 0.153 & 0.289 & 0.598 \\
\hline TS49 & Felt less masculine (most frequent) & 1.756 & 0.165 & 1.677 & 0.167 & 0.078 & 0.235 & 0.739 \\
\hline \multicolumn{9}{|c|}{ Sexual activity (SX) ${ }^{\mathrm{b}}$} \\
\hline SX50 & Sexual interest (more likely) & -0.977 & 0.334 & -1.338 & 0.315 & 0.362 & 0.459 & 0.432 \\
\hline SX51 & Sexual activity (less likely) & 0.968 & 0.344 & 1.359 & 0.330 & -0.391 & 0.477 & 0.414 \\
\hline \multicolumn{9}{|c|}{ Sexual functioning $(S X)^{b, c}$} \\
\hline SX55 & Sexual comfort (more likely) & -1.945 & 0.303 & -1.408 & 0.270 & -0.537 & 0.406 & 0.189 \\
\hline SX54 & No ejaculation problems & -0.607 & 0.228 & -0.257 & 0.220 & -0.350 & 0.316 & 0.272 \\
\hline SX53 & No erectile problems & -0.047 & 0.210 & -0.047 & 0.214 & 0.000 & 0.299 & 1.000 \\
\hline SX52 & Sexual enjoyment (less likely) & 2.441 & 0.220 & 1.888 & 0.192 & 0.552 & 0.292 & 0.061 \\
\hline
\end{tabular}

Raw responses: 1: Very much; 2: Quite a bit; 3: A little; 4: Not at all, higher score means less frequent symptom.

${ }^{b}$ Raw responses: 4: Very much; 3: Quite a bit; 2: A little; 1 : Not at all, higher score means more activity/functioning.

'Sexual functioning items are conditional and only applicable to those being sexually active. A total of 48 patients reported being sexually active in this study.

Difficulty: Item difficulty, higher difficulty means worse HRQOL/more frequent symptom.

which was consistently lower than the within-mode (paper-paper) test-retest reliability (0.61-0.93 for each item and 0.85 for total score) of the EORTC QLQ-PR25 questionnaire in another study [37]. The between-mode test-retest results may reflect the limitations of the original instrument rather than those of the data collection mode.

The equivalence testing that was used in reliability analysis was performed by two one-sided $t$-tests, which tested paired differences to be equal [38]. All the results from equivalence tests were significant indicating good equivalence, excluding Sexual functioning domain. It should be noted that of the six Sexual activity and functioning items in the EORTC QLQ-PR25, four items (SX52-SX55) are conditional and apply only to sexually active respondents. In this study, approximately half of the patients reported not being sexually active and did not respond to those 4 items, resulting in fewer responses to those conditional items in this domain. Moreover, quite a few responses were missing or showed variance in this domain due to the participants' embarrassment or reluctance to share details about their private sexual life in this study population $[39,40]$. The above reasons may have resulted in less precise measurement in the Sexual functioning domain. 
Table 5 Feasibility and preference assessments for the touch-screen mode

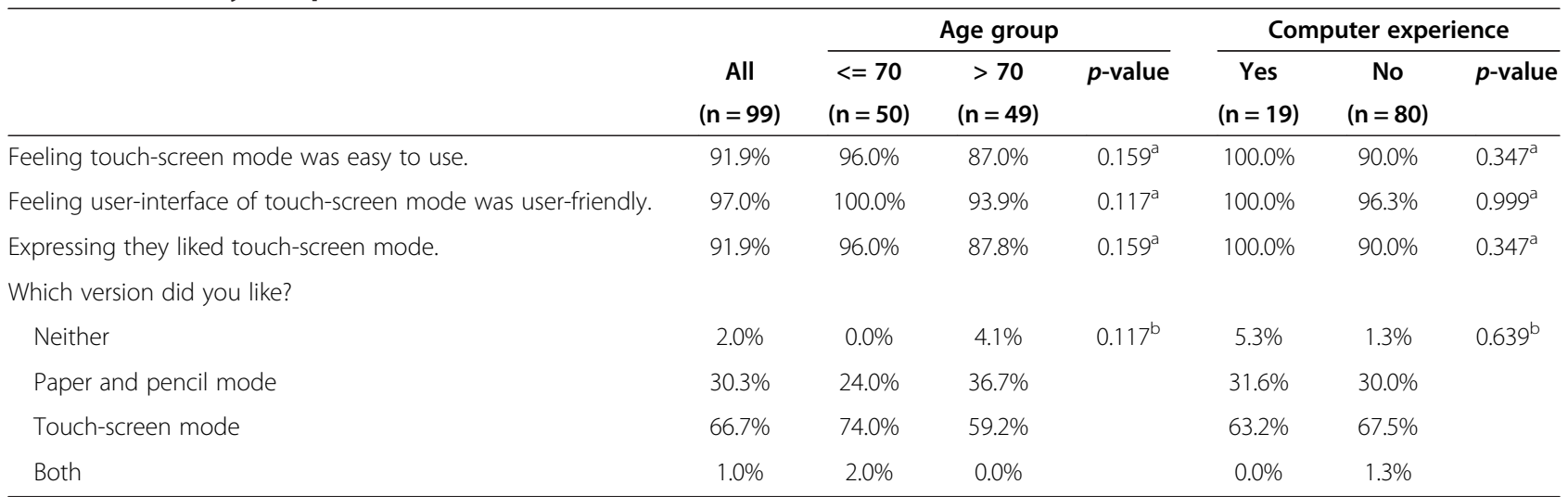

${ }^{a} p$-value was evaluated by Fisher's exact test.

${ }^{\mathrm{b}} p$-value was evaluated by Chi-square test to compare two groups: touch-screen mode and the others (three versions combined).

Moreover, DIF analysis was used to examine the difference in the difficulty papameters between two modes of administration (paper-and-pencil vs. touch-screen). Application of DIF analysis in this study allowed a thorough evaluation of measurement equivalence. We first estimated the difficulty calibration of each item for each mode separately using the rating scale model to avoid correlation problem. Then, DIF analysis was applied to assess the equivalence of item difficulties between these two modes. Although the problem of correlated data potentially existed, as the same patients in these two groups responded to the same survey twice due to the cross-over design, we mainly performed a comparison of the difficulty calibration measures, which is not so sensitive to the correlated effects of the cross-over design. For example, when performing the parallel comparison between two groups, the correlated data problem was mitigated to some extent due to the different sequence of administration, i.e., half the patients completed the questionnaire using the touch-screen mode first, followed by the paper mode, and the other half completed the paper mode first, followed by the touch-screen mode. In addition, the influence of confounding covariates and much of the betweenpatient variation could be eliminated using a crossover design, which could also increase the efficiency for estimating and testing [35,41]. DIF also benefits from this type of design.

Furthermore, there are two possible reasons to explain the inconsistency between DIF analysis and equivalence/ agreement analysis. First, these two approaches are conceptually different. DIF was used to test the scale measurement properties for each individual item between the two modes, while equivalence analysis was used to show the mean score difference of patients between two modes. Second, equivalence test as applied in this study began with a null hypothesis that the two mean values were not equivalent, and attempted to demonstrate that they were equivalent within a practical, preset limit. This test is conceptually opposite to the independent $t$-test in the DIF analysis and would lead to the result that the Sexual functioning items were more limited in equivalence/agreement, but the DIF test failed to identify this.

In this study, touch-screen administration required approximately $30 \%$ more time to complete than the paper questionnaire (20.5 $\mathrm{min}$ vs. $14.7 \mathrm{~min}$ ). We speculate that the difference in time to complete the questionnaire may be because the touch-screen/paper group was given the touch-screen version questionnaire first, and most respondents (82\%) had no experience using a computer. Furthermore, the patients may have spent more time because this was their first exposure to the EORTC QLQ-PR25 questionnaire; thus, the amount of time taken for the touch-screen mode was longer than that for the paper mode in the touch-screen/paper group (20.5 $\mathrm{min}$ vs $14.7 \mathrm{~min})(p<0.001)$. However, we found that when the touch-screen mode was performed in the second assessment, the time it took to complete the questionnaire decreased significantly $(p=0.002)$ (Table 1$)$. We anticipate that the time to complete the questionnaire via touchscreen will decrease over time as patients get used to the system. In addition, the results can be analyzed in real-time to facilitate clinical diagnosis and improve patient-physician relationships. A previous study reported that the transfer of assessment data to a computer may differentially influence the responses of older patients [42]. Previous studies of patients aged from 48.1 years to 65.0 years showed the touch-screen mode was a reliable and user-friendly method of assessing quality of life [43-46]. Our results showed that $97 \%$ of the patients reported that the touch-screen version was user-friendly and approximately $67 \%$ reported preferring the touch-screen version to the paper-and-pencil version despite the 
older average age and lack of previous experience using a computer in our study population. Moreover, most patients (92\%) reported that the touch-screen was easy to use, which was similar to the results reported by Pouwer et al. [43], which showed that the touch-screen questionnaire was easier for patients to complete even if they had rarely or never used a computer.

Patients often expect the physician to know the results of a questionnaire shortly after they have completed it; however, PROs assessed using the paper-and-pencil mode cannot be transferred to the physician's clinic in real-time. Various studies have confirmed that incorporating routine standardized HRQOL assessments in clinical oncology practice can facilitate the communication and discussion of HRQOL issues and can increase physicians' awareness of their patients' quality of life. Computer measurements are well accepted by patients who generally consider ePROs to be useful tools with which to inform their doctor about their problems [47-49].

A limitation of this study was that the washout period between the two modes of administration was only 120 minutes, which might not have been sufficiently long to completely eliminate the carry-over effects. It is possible that some residual memory or carry-over effects from the first administration were still present when the patients were asked the same set of questions during the second administration. As a result, the level of agreement between the two administrations is possibly inflated $[35,41]$. A longer interval, however, may require patients to spend too much time waiting, which might therefore discourage them from completing the second administration. Asking patients to come back on another day would likely greatly reduce the subjects' willingness to participate. Most importantly, because of the longer waiting time, the patients' condition may change and result in different answers, thereby affecting the consistency of the responses. Therefore, after careful consideration, we chose an interval of 120 minutes. Moreover, once patients had finished the first questionnaire, they were taken to the education room to watch health education videos which helped to "dilute" the memory of the first administration. Thus, we believe that the memory effect was likely lessened due to the 120-minute interval between tests and the use of health education videos.

\section{Conclusions}

Our results showed that the touch-screen mode of the Taiwan Chinese version of the EORTC QLQ-PR25 had demonstrated good reliability and was well accepted by most prostate cancer patients in Taiwan, suggesting its potential use as an alternative to the paper-and-pencil mode for measurement of PROs.

\section{Abbreviations}

EORTC: European Organization for Research and Treatment of Cancer; EORTC QLQ-PR25: EORTC quality of life questionnaire prostate-specific 25-item; HRQOL: Health-related quality of life; DIF: Differential item functioning; PROs: Patient-reported outcomes; ePROs: Electronic patient-reported outcomes; US: Urinary symptoms; BS: Bowel symptoms; TS: Hormonal treatment-related symptoms; SX: Sexual activity and function; ICC: Intraclass correlations; MCID: Minimum clinically important difference; RSM: Rating scale model.

\section{Competing interests}

All authors declare that they have no competing interests.

\section{Authors' contributions}

YJ Chang and CL Peng performed the statistical analyses and drafted the manuscript. WM Liang and $\mathrm{CH}$ Chang designed the study, wrote the protocol, and revised the manuscript. HC Wu was the coordinator of this research and conducted the field work. HC Lin, JY Wang, and TC Li participated in the design of the study, wrote the protocol, and supervised the execution of the study. YC Yeh was responsible for data collection and interpretation. All authors contributed to and approved the final manuscript.

\section{Acknowledgements}

The authors would like to thank the research support from China Medical University with project numbers CMU96-225 and CMU97-318. This study is also partially supported by the Taiwan Department of Health Clinical Trial and Research Center of Excellence (DOH99-TD-B-111-004) and the Taiwan Department of Health, China Medical University Hospital Cancer Research of Excellence (DOH99-TD-C-111-005).

\section{Author details}

${ }^{1}$ Graduate Institute of Public Health, China Medical University, Taichung, Taiwan. ${ }^{2}$ Epidemiology and Biostatistics Center, Changhua Christian Hospital, Changhua, Taiwan. ${ }^{3}$ Graduate Institute of Biostatistics, China Medical University, No.91 Hsueh-Shih Road, Taichung 40402, Taiwan. ${ }^{4}$ Buehler Center on Aging, Health \& Society, Northwestern University Feinberg School of Medicine, Chicago, IL, USA. ${ }^{5}$ Department of Urology, China Medical University Hospital, Taichung, Taiwan. ${ }^{6}$ School of Medicine, China Medical University, Taichung, Taiwan. ${ }^{7}$ Department of Health Risk Management, China Medical University, Taichung, Taiwan. ${ }^{8}$ Department of Health Services Administration, China Medical University, Taichung, Taiwan. ${ }^{9}$ Biostatistics Center, China Medical University, Taichung, Taiwan.

Received: 4 July 2013 Accepted: 14 February 2014

Published: 20 February 2014

\section{References}

1. Chang $\mathrm{CH}$ : Patient-reported outcomes measurement and management with innovative methodologies and technologies. Qual Life Res 2007, 16(Suppl 1):157-166.

2. Gwaltney CJ, Shields AL, Shiffman S: Equivalence of electronic and paper-and-pencil administration of patient-reported outcome measures: a meta-analytic review. Value Health 2008, 11:322-333.

3. Bennett AV, Jensen RE, Basch E: Electronic patient-reported outcome systems in oncology clinical practice. CA Cancer J Clin 2012, 62:337-347.

4. Young NL, Varni JW, Snider L, McCormick A, Sawatzky B, Scott M, King G, Hetherington R, Sears E, Nicholas D: The Internet is valid and reliable for child-report: an example using the Activities Scale for Kids (ASK) and the Pediatric Quality of Life Inventory (PedsQL). J Clin Epidemiol 2009, 62:314-320.

5. Luckett T, Butow PN, King MT: Improving patient outcomes through the routine use of patient-reported data in cancer clinics: future directions. Psychooncology 2009, 18:1129-1138.

6. Coons SJ, Gwaltney CJ, Hays RD, Lundy JJ, Sloan JA, Revicki DA, Lenderking WR, Cella D, Basch E: Recommendations on evidence needed to support measurement equivalence between electronic and paper-based patient-reported outcome (PRO) measures: ISPOR ePRO Good Research Practices Task Force report. Value Health 2009, 12:419-429.

7. de Bree R, Verdonck-de Leeuw IM, Keizer AL, Houffelaar A, Leemans CR: Touch screen computer-assisted health-related quality of life and distress data collection in head and neck cancer patients. Clin Otolaryngol 2008, 33:138-142. 
8. Mullen $\mathrm{KH}$, Berry DL, Zierler BK: Computerized symptom and quality-of-life assessment for patients with cancer part II: acceptability and usability. Oncol Nurs Forum 2004, 31:E84-E89.

9. Matthew AG, Currie KL, Irvine J, Ritvo P, Santa Mina D, Jamnicky L, Nam R, Trachtenberg J: Serial personal digital assistant data capture of health-related quality of life: a randomized controlled trial in a prostate cancer clinic. Health Qual Life Outcomes 2007, 5:38.

10. Salaffi F, Gasparini S, Grassi W: The use of computer touch-screen technology for the collection of patient-reported outcome data in rheumatoid arthritis: comparison with standardized paper questionnaires. Clin Exp Rheumatol 2009, 27:459-468.

11. Bureau of Health Promotion, Department of Health, Executive Yuan, Taiwan: Taiwan cancer registry annual report. Taipei: Taiwan Cancer Registry; 2013.

12. Chie WC, Yu CC, Yu HJ: Reliability and validity of the Taiwan Chinese version of the EORTC QLQ-PR25 in assessing quality of life of prostate cancer patients. Urol Sci 2010, 21:118-125.

13. van Andel G, Bottomley A, Fossa SD, Efficace F, Coens C, Guerif S, Kynaston H, Gontero P, Thalmann G, Akdas A, et al: An international field study of the EORTC QLQ-PR25: a questionnaire for assessing the health-related quality of life of patients with prostate cancer. Eur J Cancer 2008, 44:2418-2424.

14. Arraras JI, Villafranca E, Arias de la Vega F, Romero P, Rico M, Vila M, Asin G, Chicata V, Dominguez MA, Lainez N: The EORTC Quality of Life Questionnaire for patients with prostate cancer: EORTC QLQ-PR25. Validation study for Spanish patients. Clin Trans/ Oncol 2009, 11:160-164.

15. Chang YJ, Liang WM, Wu HC, Lin HC, Wang JY, Li TC, Yeh YC, Chang CH: Psychometric evaluation of the Taiwan Chinese version of the EORTC QLQ-PR25 for HRQOL assessment in prostate cancer patients. Health Qual Life Outcomes 2012, 10:96.

16. Fayers PM, Aaronson NK, Bjordal K, Groenvold M, Curran D, Bottomley A: The EORTC QLQ-C30 Scoring Manual. 3rd edition. Brussels: European Organization for Research and Treatment of Cancer; 2001.

17. Lin HC, Wu HC, Chang CH, Li TC, Liang WM, Wang JY: Development of a real-time clinical decision support system upon the Web MVC-based architecture for prostate cancer treatment. BMC Med Inform Decis Mak 2011, 106:249-259.

18. Velikova G, Wright EP, Smith AB, Cull A, Gould A, Forman D, Perren T, Stead M, Brown J, Selby PJ: Automated collection of quality-of-life data: a comparison of paper and computer touch-screen questionnaires. J Clin Oncol 1999, 17:998-1007.

19. Bartko JJ: On various intraclass correlation reliability coefficients. Psychol Bull 1976, 83:762-765.

20. Stokdijk M, Biegstraaten M, Ormel W, de Boer YA, Veeger HE, Rozing PM: Determining the optimal flexion-extension axis of the elbow in vivo - a study of interobserver and intraobserver reliability. J Biomech 2000, 33:1139-1145.

21. Limentani GB, Ringo MC, Ye F, Berquist ML, McSorley EO: Beyond the t-test: statistical equivalence testing. Anal Chem 2005, 77:221A-226A

22. Tunes Da Silva G, Logan BR, Klein JP: Methods for equivalence and noninferiority testing. Biol Blood Marrow Transplant 2009, 15:120-127.

23. Kong L, Kohberger RC, Koch GG: Type I error and power in noninferiority/ equivalence trials with correlated multiple endpoints: an example from vaccine development trials. J Biopharm Stat 2004, 14:893-907.

24. Greene CJ, Morland LA, Durkalski VL, Frueh BC: Noninferiority and equivalence designs: issues and implications for mental health research. J Trauma Stress 2008, 21:433-439.

25. Osoba D, Rodrigues G, Myles J, Zee B, Pater J: Interpreting the significance of changes in health-related quality-of-life scores. J Clin Oncol 1998, 16:139-144.

26. Maringwa JT, Quinten C, King M, Ringash J, Osoba D, Coens C, Martinelli F, Vercauteren J, Cleeland CS, Flechtner $\mathrm{H}$, et al: Minimal important differences for interpreting health-related quality of life scores from the EORTC QLQ-C30 in lung cancer patients participating in randomized controlled trials. Support Care Cancer 2011, 19:1753-1760.

27. Pickard AS, Dalal MR, Bushnell DM: A comparison of depressive symptoms in stroke and primary care: applying Rasch models to evaluate the center for epidemiologic studies-depression scale. Value Health 2006, 9:59-64.

28. Conrad KJ, Smith EV Jr: International conference on objective measurement: applications of Rasch analysis in health care. Med Care 2004, 42:11-16.

29. Embretson SE, Reise SP: Item Response Theory for Psychologists. Hillsdale, NJ: Lawrence Erlbaum Associates; 2000.
30. Hambelton RK, Swaminathan H, Rogers HJ: Fundamentals of Item Response Theory. London: Sage; 1991.

31. Bond TG, Fox CM: Applying the Rasch Model: Fundamental Measurement in the Human Sciences. Hillsdale, NJ: Lawrence Erlbaum Associates; 2001.

32. Bushnell DM, Martin ML, Parasuraman B: Electronic versus paper questionnaires: a further comparison in persons with asthma. J Asthma 2003, 40:751-762.

33. The EORTC Quality of Life Groups' Website. [http://groups.eortc.be/qol/ faq?tid=12]. Brussels: European Organisation for Research and Treatment of Cancer; Accessed January 20, 2014.

34. US Food and Drug Administration: Guidance for Industry, on Patient-Reported Outcome Measures: Use in Medical Product Development to Support Labeling Claims. Rockville, MD: U.S: Department of Health and Human Services, Food and Drug Administration; 2009.

35. Mills EJ, Chan AW, Wu P, Vail A, Guyatt GH, Altman DG: Design, analysis, and presentation of crossover trials. Trials 2009, 10:27.

36. Maclure $M$ : The case-crossover design: a method for studying transient effects on the risk of acute events. Am J Epidemiol 1991, 133:144-153.

37. Hejazi J, Rastmanesh R, Taleban FA, Molana SH, Ehtejab G: A pilot clinical trial of radioprotective effects of curcumin supplementation in patients with prostate cancer. J Cancer Sci Ther 2013, 5:320-324.

38. Steffen $\mathrm{T}$, Seney M: Test-retest reliability and minimal detectable change on balance and ambulation tests, the 36-item short-form health survey, and the unified Parkinson disease rating scale in people with parkinsonism. Phys Ther 2008, 88:733-746.

39. Hwang HF, Liang WM, Chiu YN, Lin MR: Suitability of the WHOQOL-BREF for community-dwelling older people in Taiwan. Age Ageing 2003, 32:593-600.

40. Wang TF, Lu CH, Chen IJ, Yu S: Sexual knowledge, attitudes and activity of older people in Taipei, Taiwan. J Clin Nurs 2008, 17:443-450.

41. Wang D, Bakhai A: In Clinical Trials: A Practical Guide to Design, Analysis and Reporting. Edited by Wang D, Bakhai A. London: Remedica; 2006:91-99.

42. Bowling A: Mode of questionnaire administration can have serious effects on data quality. J Public Health (Oxf) 2005, 27:281-291.

43. Pouwer F, Snoek FJ, van der Ploeg HM, Heine RJ, Brand AN: A comparison of the standard and the computerized versions of the Well-being Questionnaire (WBQ) and the Diabetes Treatment Satisfaction Questionnaire (DTSQ). Qual Life Res 1998, 7:33-38.

44. Kleinman L, Leidy NK, Crawley J, Bonomi A, Schoenfeld P: A comparative trial of paper-and-pencil versus computer administration of the Quality of Life in Reflux and Dyspepsia (QOLRAD) questionnaire. Med Care 2001, 39:181-189.

45. Greenwood MC, Hakim AJ, Carson E, Doyle DV: Touch-screen computer systems in the rheumatology clinic offer a reliable and user-friendly means of collecting quality-of-life and outcome data from patients with rheumatoid arthritis. Rheumatology 2006, 45:66-71.

46. Millsopp L, Frackleton S, Lowe D, Rogers SN: A feasibility study of computer-assisted health-related quality of life data collection in patients with oral and oropharyngeal cancer. Int J Oral Maxillofac Surg 2006, 35:761-764.

47. Detmar SB, Muller MJ, Schornagel JH, Wever LD, Aaronson NK: Health-related quality-of-life assessments and patient-physician communication: a randomized controlled trial. JAMA 2002, 288:3027-3034.

48. Henderson A, Andreyev HJ, Stephens R, Dearnaley D: Patient and physician reporting of symptoms and health-related quality of life in trials of treatment for early prostate cancer: considerations for future studies. Clin Oncol 2006, 18:735-743.

49. Velikova G, Brown JM, Smith AB, Selby PJ: Computer-based quality of life questionnaires may contribute to doctor-patient interactions in oncology. Br J Cancer 2002, 86:51-59.

doi:10.1186/1477-7525-12-23

Cite this article as: Chang et al:: Measurement equivalence and feasibility of the EORTC QLQ-PR25: paper-and-pencil versus touch-screen administration. Health and Quality of Life Outcomes 2014 12:23. 